\title{
Proceso productivo de empresas queseras artesanales de la región Ríos de Tabasco
}

\author{
Liliana Cristel Blanco Gómez - Ana Laura Luna Jiménez ${ }^{2}$ - Nicolás González Cortés ${ }^{3}$ - \\ Mateo Ortiz Hernández $z^{4}$ - Román Jiménez Vera ${ }^{5}$
}

Recepción: 01/11/2018 Aceptación: 23/03/2019

\begin{abstract}
Resumen
Los Ríos es una de las cinco regiones productivas de Tabasco, México, donde la ganadería es la principal actividad económica; y la quesería, la mayor actividad de transformación. El objetivo fue conocer el proceso productivo de las empresas queseras artesanales ubicadas en esta región. Se recabó información mediante un cuestionario relacionado con el diseño del proceso, producción y distribución de productos.
\end{abstract}

Las queserías de la región Ríos son microempresas familiares con inversión de capital privado. Su producción se enfoca a la elaboración de productos de consumo local, principalmente el queso de poro. Aunque se cuenta con una asociación, las empresas laboran de manera individual y no cuentan con procesos documentados ni estandarizados.

La producción se distribuye a mercados locales; muy pocas a nivel nacional y ninguna en el ámbito internacional. El gobierno mexicano ha apoyado el desarrollo mediante leyes y eventos. No obstante, las empresas deben delimitar las características de sus productos, desde la materia prima hasta su distribución. De igual manera, es indispensable mejorar los procesos que involucren la inocuidad, el empaque, condiciones de conservación, estandarización y documentación de procesos.

Palabras clave: Queso, empresa familiar, artesanal, ganadería, queso de poro.

\section{Abstract}

Los Rios is one of the five productive region of Tabasco, Mexico, where cattle ranching is the main economic activity; and cheese making is the main transformation activity. The objective was to know the productive process of the artisan cheese companies located in this region. The Information was collected through a questionnaire related to process design, production and distribution of products.

The cheese factories in Rios region are micro family businesses with private capital investment. Its production focuses of the production of local production consumer products, mainly pore cheese. Although there is an association, companies work individually and do not have documented or standardized processes.

Production is distributed to local markets; very few at the national level and none at the international level. The Mexican government has supported development through laws and events. However, companies must define the characteristics of their products, from raw material to distribution. Similarly, it is essential to improve the processes involving safety, packaging, conservation conditions, standardization and documentation of processes.

Key words: Cheese, family business, handicraft, livestock, pore cheese.

1. Egresada del programa educativo Ingeniería en Alimentos, impartido en la Universidad Juárez Autónoma de Tabasco, División Académica Multidisciplinaria de los Ríos, México.

2. Licenciada en Administración, Profesora de los programas educativos y Miembro del Cuerpo Académico Desarrollo Sustentable; Universidad Juárez Autónoma de Tabasco, División Académica Multidisciplinaria de los Ríos, México.

3. Ingeniero en Alimentos, Profesor de los programas educativos y Miembro del Cuerpo Académico Desarrollo Sustentable; Universidad Juárez Autónoma de Tabasco, División Académica Multidisciplinaria de los Ríos, México.

4. Maestría en Desarrollo Agropecuario Sustentable, Profesor de los programas educativos y Miembro del Cuerpo Académico Desarrollo Sustentable; Universidad Juárez Autónoma de Tabasco, División Académica Multidisciplinaria de los Ríos, México.

5. Doctorado en Biotecnología y Doctorado en Alta Dirección, Profesor Investigador, Universidad Juárez Autónoma de Tabasco, División Tenosique, Tabasco-México. C. P. 86901; Autor para correspondencia: email: roman.jimenez@ujat.mx 


\section{Introducción}

Los Ríos es una de las cinco subregiones productivas que conforman el estado de Tabasco, México (Chontalpa, Centro, Sierra, Ríos y Pantanos). Esta región tiene una extensión que corresponde al 24.65\% del estado, compuesto por tres municipios: Tenosique, Balancán y Emiliano Zapata. La principal actividad económica de esta zona es la agropecuaria, sobresaliendo la ganadería y la agricultura (Estrada et al., 2013; Capdepont-Ballina y Marín-Olán, 2014).

La producción lechera se realiza en ranchos ganaderos, los cuales han desarrollado el sistema vaca-becerro, seguido del sistema de rejeguería tradicional. Los ranchos poseen alrededor de 37 cabezas de ganado, con nivel tecnológico bajo y fuerza de trabajo familiar (Manjarrez et al., 2007).

La región Ríos es llamada así por la gran cantidad de ríos que la cruzan, entre ellos, el río Usumacinta, el más caudaloso del país. Esta región está localizada en una superficie de territorio casi plana, con ondulaciones inferiores a 40m.s.n.m., y abarca la red hídrica que forma parte del sistema Grijalva-Usumacinta (ver figura 1).

La precipitación pluvial es de 2,343mm anuales y se conservan zonas selváticas; aunque predominan sabanas y pastizales (Barba-Macías et al., 2006). La temperatura media men- sual oscila entre 22 y $28^{\circ} \mathrm{C}$ y la precipitación fluctúa de 1,800 a 2,500mm anuales (Manjarrez et al., 2007).

Con la globalización, la industrialización y homogenización de productos han tomado auge en la producción y consumo a nivel mundial. Los alimentos producidos localmente $-\mathrm{y}$ de manera artesanal- no pueden competir en costos y volumen de producción con las grandes firmas internacionales. Estos productos locales son relegados a mercados regionales por las limitaciones para su comercialización y por una producción insuficiente (Ayuso y Castillo, 2017).

En la medida que la globalización tiende a homogeneizar los productos a nivel mundial, se plantea el gran reto para América Latina: el de añadir valor agregado a las manufacturas, artesanías y alimentos obtenidos de manera artesanal. La globalización económica también significa diversificación de mercados y los productos específicos, con denominación de origen, con particularidades culturales, que los hacen diferentes a los productos industriales; también tienen una acogida extraordinaria y hasta un sobreprecio en los mercados mundiales (Barragán y Ovando, 2015).

Los pequeños productores de lácteos cuentan con la ventaja de participar en las cadenas agroalimentarias de producción de quesos artesanales, ya que funcionan como proveedores de la materia prima requerida 


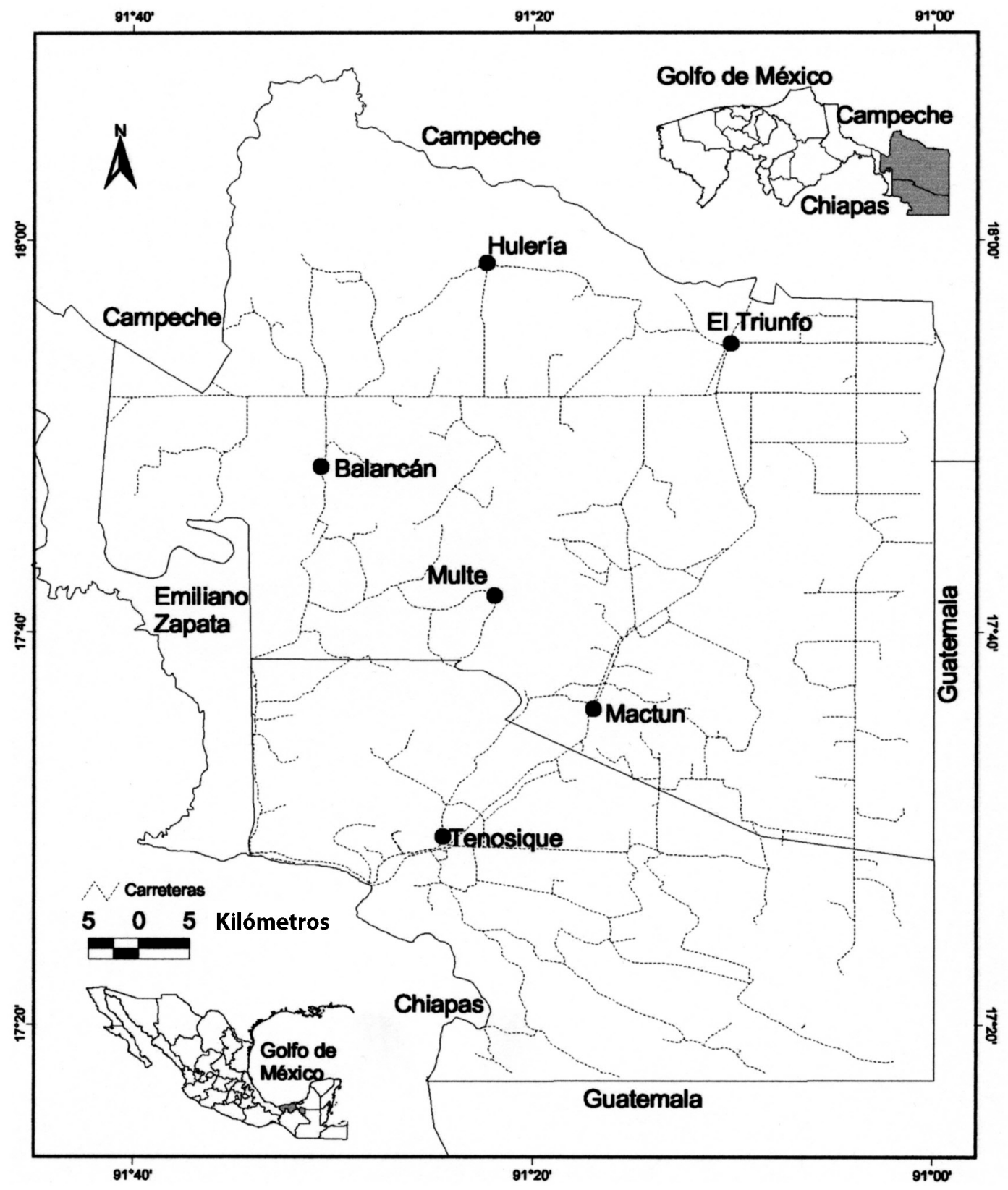

Figura 1. Localización de la subregión Ríos, Tabasco, México.

Fuente: Manjarrez et al., 2007.

o ellos mismos realizan las dos actividades como lecheros y queseros (Villegas y Cervantes, 2011).

En Tabasco, las micros y pequeñas empresas son las encargadas de realizar la transfor- mación y procesamiento de materias primas agropecuarias, donde la empresa familiar representa alrededor del 90\% de los establecimientos industriales. Actualmente, estas empresas enfrentan el reto de la modernización, 
por lo que deben adecuarse a procesos que les permitan prepararse para responder a los desafíos de la nueva economía, basada en el conocimiento (Salvador-Valencia y Reyes-De la Cruz, 2011).

La productividad y la competitividad son criterios que pueden ser áreas de oportunidad. Para algunas organizaciones estos conceptos pueden encontrarse en pugna; para otras, representan la opción de aplicarse en la búsqueda del crecimiento. La competitividad puede llevarlos a la creación de productos únicos, especiales, necesarios y de valor diferenciado que les produzca la posición de producto competitivo, logrando especial importancia en las estrategias de diferenciación y segmentación (Castellot, 2014).

Otro reto que enfrentan las empresas queseras artesanales es el desconocimiento de sus productos y sus cualidades. En México, la situación de los quesos artesanales es preocupante, debido que son desconocidos por la mayoría de la población y no existe investigación científica que apoye sus propiedades (Queso de Poro Genuino de Balancán, 2009).

Los quesos artesanales generalmente son de circulación local o regional; tienen como nichos de mercado a consumidores de esos mismos espacios geográficos $\mathrm{y}$, recientemente, a una creciente población de clientes que busca productos de calidad con evocación de lo tradicional y genuino, pero respetando las tradiciones locales y el medio ambiente. La quesería artesanal reviste gran relevancia por su capacidad para generar y mantener el empleo rural hacia un gran número de agentes de la cadena agroindustrial de la leche, como ganaderos, queseros y comerciantes (Villegas y Cervantes, 2011).

Los estudios relacionados con la valorización de las actividades de la agroindustria rural de producción de quesos en México son indispensables, debido a la participación relevante económica y social que tienen en el país (Castañeda et al., 2009). El objetivo de este trabajo fue conocer el proceso productivo de las empresas queseras de la región Ríos de Tabasco, México, con la finalidad de mejorar su competitividad.

\section{Materiales y Métodos}

La unidad de análisis estuvo conformada por las queserías localizadas en la subregión Ríos del estado de Tabasco en México. De un padrón de 35 empresas registradas en la zona, 19 aceptaron participar en el estudio: diez del municipio de Balancán y nueve del municipio de Tenosique. Se realizó un estudio exploratorio-descriptivo cuyo diseño metodológico responde a un enfoque de tipo social, donde se obtuvo la información a partir de la fuente primaria: las empresas queseras de la región.

Los datos fueron recopilados a través de encuesta junto con la observación directa y 
participativa de los investigadores, lo que permitió identificar y caracterizar agentes y describir relaciones en el proceso estudiado. La encuesta se aplicó a través de una entrevista personal a los dueños o encargados de la empresa, mediante un instrumento de medición, que consistió en un cuestionario estructurado en seis dimensiones (datos generales de la empresa, materia prima, proceso, control de calidad, mercados y vinculación) con 22 ítems totales.

\section{Resultados y Discusión}

La región Ríos de Tabasco, México, es una zona primordialmente agropecuaria, siendo la característica de sus suelos ácidos lo que favoreció el cultivo de pastizales e impulsó la ganadería. Hoy, la actividad ganadera es la principal fuente de ingresos de la región (Cano et al., 2011).

Este trabajo se realizó con la finalidad de analizar el proceso productivo de las empresas queseras establecidas en la zona. Se encontró que la mayoría de ellas son microempresas, iniciadas con capital privado familiar donde prevalece la experiencia transmitida de una generación a otra. Esta clasificación se realizó tomando en cuenta el número promedio de trabajadores: el 83\% de las empresas proporciona empleo a menos de diez personas, lo que muestra que la mayoría de estas queserías son micros, donde se contrata principalmente a familiares.
De acuerdo con los entrevistados, en la contratación de personal se da preferencia a parientes, aunque no muestren alto conocimiento del "saber hacer". El 17\% restante de las empresas son pequeñas, ya que el número de sus empleados es mayor a diez, pero menor a cincuenta.

En cuanto al diseño del proceso, solo las grandes empresas del sector lácteo involucran la participación de diferentes actores económicos en la producción, transformación, transporte, almacenamiento, financiamiento, regulación y comercialización de sus productos (Castañeda et al., 2009). Estas empresas integran el proceso agroindustrial desde la producción de la materia prima hasta el consumo, y pueden operar con leche fresca, en polvo u otros insumos lácteos; mientras que las micros y pequeñas, solo procesan materia prima fresca y local.

La presencia de micros en la región Ríos contrasta con otras regiones del país, donde predominan empresas de mayor tamaño. En un estudio realizado por Castañeda et al. (2009) relacionado con la concentración de agroindustrias rurales de producción de quesos en el noroeste del Estado de México, se encontró que entre 1960 y 1980, solo existían seis empresas que producían quesos. Sin embargo, en los años noventa se incorporaron 19 agroindustrias y, nueve más al iniciar la presente década. 
Dentro de su estructura coexisten grandes empresas en número reducido, con un gran número de pequeñas agroindustrias con problemas para articularse al mercado y de calidad en sus productos. En la región Ríos, aunque es una zona ganadero-lechera, no existen empresas de gran tamaño, solo micro y pequeñas.

Boscán et al. (2007) reportaron que, dentro de las empresas del sector de derivados lácteos de Zulia, Venezuela, específicamente respecto al número promedio de trabajadores anuales, el 25\% de ellas proporciona empleo a menos de diez personas. Un 62.5\% de los casos analizados contrata entre once y cincuenta personas, en tanto que, solo el $12.5 \%$, emplea entre 51 y 100 trabajadores. Estos datos muestran la necesidad de crecimiento de las empresas queseras de la subregión Ríos, ya que cerca del $90 \%$ corresponde a microempresas.

En el año 2002, con la finalidad de incrementar la competitividad de las empresas mexicanas, el gobierno implementó diversas medidas y estrategias como la creación de la Ley para el Desarrollo de la Competitividad de la Micro y Pequeña Empresa. Luego, en el año 2004 se creó el Instituto Mexicano para la Competitividad; y en 2009, el Primer Foro para la Competitividad en México. Estas acciones buscan alcanzar un mejor desempeño económico de las empresas, centradas en el fortalecimiento del estado de derecho; mejores condiciones para la inversión y la crea- ción de empleos, igualdad de oportunidades educativas, profesionales y de salud; gobernabilidad democrática, política exterior para alcanzar niveles de crecimiento y bienestar (Patlán, 2013).

En la región Ríos de Tabasco no existen empresas grandes dedicadas a la producción quesera. Esto probablemente se relacione con que en el estado se desarrolla primordialmente la ganadería de doble propósito, considerada como un sistema tradicional de producción en la región tropical, donde mediante cruzamientos de razas se obtienen simultáneamente los productos leche y carne (Arce et al., 2017). Además, sus trabajos se enfocan en procesar el excedente de la producción lechera como una actividad complementaria a la comercialización de la carne.

La etapa de elaboración, dentro del proceso productivo, se caracteriza por la transformación de las materias primas hasta que obtener el producto o servicio final. La quesería artesanal reviste gran relevancia, no solamente porque elabora un producto de reconocidas bondades nutricionales y gustativas, sino por su capacidad para generar y mantener el empleo rural para un gran número de agentes de la cadena agroindustrial.

Las queserías de la región definen sus productos de acuerdo al mercado regional. Entre los principales productos que elaboran destacan los quesos de poro, tipo Oaxaca, crema, 
sopero, tipo Cotija, panela, fresco, botanero y la mantequilla. Estos resultados son similares a los presentados por Castro-Georgana et al. (2005), quienes mencionan que, a nivel estatal, los principales quesos elaborados son: fresco, crema, doble crema, tipo Oaxaca, tipo Cotija, tipo chihuahua, botanero, de poro $y$ tipo manchego.

El 55.6\% de las empresas de la zona de los Ríos afirma que su producto líder es el queso de poro, seguido por el queso tipo Oaxaca y la mantequilla. En un estudio realizado en el estado de Zulia, en Venezuela, Boscán et al. (2007) caracterizaron a las empresas procesadoras de derivados lácteos, encontrando como principales productos la crema de leche (nata) mantequilla y queso diversos como mozzarella, ricota, requesón, de mano, palmita, blanco pasteurizado y blanco duro; la mayoría elaborados con tecnología importada.

Cada empresa presenta un producto, conocido como producto líder, que sobresale en cuanto al porcentaje de ventas anuales. De igual manera, las empresas queseras de la región Ríos producen diversos tipos de queso, $\mathrm{y}$ tienen como producto líder el queso conocido como queso de poro o queso de Balancán (ver figura 2).

El queso de poro es el único en Tabasco que cuenta con una marca colectiva autorizada por el Instituto Mexicano de la Protección Industrial (IMPI). Este queso es producido por pequeñas empresas de los municipios de Emiliano Zapata, Balancán y Tenosique que conforman la Sociedad de Productores de Queso de Poro Genuino en la subregión Ríos.

En un estudio realizado por Hernández et al. (2011) sobre el proceso socio-técnico de producción del queso añejo de Zacazonapan, en el Estado de México, se encontró que el sistema agroindustrial presenta estructuras colectivas únicamente en la fase primaria de producción y en la forma de organizaciones de productores; mientras que los queseros muestran una actitud individualista y de desconfianza; siendo la cadena agroindustrial coordinada verticalmente. A diferencia de estos, los productores de queso de la subregión Ríos se han unido para establecer una marca colectiva.

El propósito de establecer la marca colectiva fue generar estrategias que deriven en un incremento de la valorización económica y posicionamiento en el mercado. Se trata de un queso mexicano genuino $y$, al igual que otros quesos artesanales, se vende de manera local-regional y carece de una estrategia de mercado que sea soportada en estudios objetivos. Este es el quinto queso en México que logra dicha distinción junto con el queso Cotija (Michoacán), queso de bola (Chiapas), queso crema (Chiapas) y Queso de Tepalcatepec (Michoacán). Lo anterior representa un gran paso para la revalorización de productos 


\begin{tabular}{|l|l|l}
\hline Producto & Ejemplo & \multicolumn{1}{c}{ Descripción } \\
\hline Queso de poro & $\begin{array}{l}\text { Es un queso con un aroma intenso, sabor ácido- } \\
\text { salado. Desarrolla en el interior de la pasta } \\
\text { pequeños poros por acumulación de gas } \\
\text { generado por microorganismos autóctonos, en } \\
\text { gran medida provenientes de la leche y del } \\
\text { suero utilizados en su fabricación. }\end{array}$
\end{tabular}

Figura 2. Queso de poro elaborado en la región de Ríos, México.

elaborados con procedimientos tradicionales (Cervantes et al., 2017).

Aunque se ha avanzado en la marca colectica del queso de poro, las empresas integrantes de la marca no han delimitado el uso del nombre, ya que empresas que no forman parte de la sociedad productora emplean este nombre. Se sugiere un control sobre el uso del mismo, ya que empresas con procesos no estandarizados, pueden interferir en la calidad y la percepción de las características del queso de poro, resultando en un rechazo por parte de los consumidores locales y nacionales.

En México existe un predominio de los quesos frescos. La producción de queso tipo $\mathrm{Oa}-$ xaca ha ido en aumento, mientras que la producción de queso maduro se estancó; incluso disminuyó la producción de queso manchego. Esta situación revela las estrategias de las agroindustrias queseras mexicanas, que se enfocan en los quesos frescos, por ser los más consumidos, los más fáciles de hacer, y los más rentables (Pomeón y Cervantes, 2010).

En cuanto a los mercados, la mayoría de las empresas de la región Ríos cuenta con pun- tos de ventas locales y regionales, principalmente en los municipios cercanos como Balancán, Tenosique, Macuspana y la ciudad de Villahermosa, en Tabasco; Morelos y la Ciudad de México a nivel nacional. La venta se realiza al menudeo en expendios propios y en tiendas de abarrotes locales. Es importante para las microempresas de quesos en la región buscar nuevos mercados para la distribución de sus productos, así como formas efectivas de dar a conocerlos.

Con la intención de impulsar la producción de quesos artesanales de la región y promover el producto de las empresas locales hacia otros mercados, desde el año 2013, el gobierno del estado de Tabasco, en coordinación con los municipios de la región, realiza el Festival del Queso Artesanal. Este festival promueve la industria turística, cultural y gastronómica del estado, particularmente la de los municipios de la subregión Ríos. Las empresas queseras tienen la oportunidad de mostrar sus productos para ampliar la comercialización de sus productos entre los visitantes al evento. En un estudio realizado por Cervantes et al. (2016), al evaluar el valor simbólico del queso de poro, se encontró que su consumo está 
asociado a sensaciones relacionadas con recuerdos de lugares (42\%), a momentos (25\%) y a algún familiar, familia u otras personas cercanas como amigos; así como a algún momento específico de su vida (26\%). Por lo que, las empresas que producen alimentos nostálgicos o de añoranza pueden aprovechar los potenciales mercados de destino para promocionar sus productos.

De acuerdo con Villegas y Cervantes (2011), los quesos artesanales generalmente son de circulación local o regional y tienen como nichos de mercado a consumidores de esos mismos espacios geográficos. Pero recientemente está surgiendo una creciente población de clientes que buscan productos lácteos de calidad, con evocación de lo tradicional y genuino; encabezando esta tendencia, los migrantes que habitan en otros estados o fuera del país.

Una fortaleza de las queserías de la región Ríos es que la materia prima para la elaboración de quesos es producida por la propia empresa; y en periodos de sequía, cuando la producción de leche disminuye, se compra a productores de la región, quienes se ubican en la misma comunidad que las empresas transformadoras. Estas comercializan directamente la producción y trasportan la materia prima a las queserías.

Los productores de queso adquieren la materia prima; no obstante, $30 \%$ cuenta con hatos lecheros y su producción es canalizada para complementar el requerimiento diario de la empresa. Cada empresa adquiere la materia prima de manera distinta.

El 50\% se abastece de productores de leche y producción propia; por su parte, el $20 \%$ recurre solo a productores, mientras que ninguna empresa transforma exclusivamente la producción de su hato. En el 2009, con el cierre de la empresa procesadora de leche Ultralácteos ${ }^{\circledast}$, los productores perdieron un importante canal de comercialización, por lo que una opción fue la venta de leche a microempresas queseras de la región (Rubio et al., 2012).

Correa et al. (2006) analizaron casos de estudio de diversos países de América Latina, y describieron la organización de las empresas en el territorio y clasificaron las zonas de producción de la materia prima como integradas, semi-integradas y separadas. Las empresas queseras de la subregión Ríos se consideran semi-integradas por la ubicación en comunidades específicas y porque el abasto de leche deriva de productores localizados en la misma localidad que las empresas y de hatos propios. Conocer el origen de la materia prima puede apoyar a mantener la calidad mediante la implementación de buenas prácticas de producción, ya que muchos de los productos artesanales locales se realizan con leche no pasteurizada (Castro-Georgana et al., 2005). 
La mayoría de las empresas queseras de la región no realiza análisis de control de calidad, ni a la leche, ni al producto terminado. Sus procesos no se encuentran documentados, aunque algunas empresas expresaron que algunos de sus procesos los tienen estandarizados. Estos datos muestran una tendencia similar en todo el estado de $\mathrm{Ta}$ basco, ya que Castro-Georgana et al . (2005), reportaron que en catorce municipios de Tabasco se cuenta con un padrón de 112 fábricas de quesos; de las cuales solamente el $1.8 \%$ pasteuriza la leche. Esto corresponde a solo dos establecimientos del municipio del centro del estado.

En un estudio realizado por Díaz et al. (2016), en dos empresas productoras de queso de poro se encontró que las plantas cumplen con el 20 y el 70\% de los parámetros establecidos por la regulación vigente sobre el cumplimiento de Buenas Prácticas de Manufactura (BPM) para elaboración de queso de poro. Estos resultados muestran que la implementación de sistemas más robustos como HACCP (Hazard Analysis and Critical Control Points) aún no es factible.

Se muestra con ello la necesidad de capacitación para los productores en aras de cumplir con los requerimientos de personal y la adecuación de equipos y utensilios; mientras que el apoyo financiero podría mejorar el rubro de los requerimientos de las instalaciones y también el de equipos y utensilios.
En cuanto al empaque de los quesos, la mayoría emplea cualquier tipo de cera (59\%), sin ser de grado alimenticio; seguido por el empaque al vacío (25\%) como nueva tendencia; aunque no se han realizado estudios sobre el impacto en la microflora de los quesos, así como el perfil sensorial y toxicológico. El 8\% utiliza bolsas de plástico y un sector (8\%) comercializa sus productos sin empaque.

Actualmente, el empaque de los quesos se ha diversificado, empleando material flexible, ya que los consumidores hacen mayores exigencias respecto a los empaques de los productos lácteos, entre sus exigencias están el uso de empaques transparentes para apreciar mejor el producto antes de comprarlo (Vargas-Uscategui et al., 2017).

Por otra parte, el uso de materiales biodegradables presenta una alternativa al uso de materiales plásticos. Los recubrimientos comestibles a partir de polisacáridos se han convertido en una opción atractiva y rentable para la conservación de alimentos. Tapiero et al . (2017) evaluaron películas de carboximetilcelulosa, incorporando glicerol como plastificante y aceite esencial de jengibre (Zingiber officinale) como agente antimicrobiano para la conservación de quesos semimaduros tipo paipa. Este empaque, además de conservar el producto, logró reducir la carga microbiana para el producto final, convirtiéndose en una alternativa agroindustrial para la conservación de quesos. 
En periodos de abundancia de leche, cuando se requiere contratar mayor personal o para cubrir puestos de trabajo, la mayoría de las queserías (75\%) emplea familiares, seguido por personas que sepan el oficio. Las empresas mencionan que sus ganancias no les permiten contratar personal universitario. Sin embargo, el 50\% de ellas estuvo de acuerdo en participar en programas de vinculación universitarios, así como en la bolsa de trabajo.

Las actividades organizativas son un elemento positivo para la concentración de empresas, si se considera la dificultad que presentan para mejorar la calidad y comercialización de los productos lácteos. Los estudios relacionados con la valorización de las actividades de la agroindustria rural de producción de quesos en México son indispensables (Castañeda et al., 2009).

Los quesos artesanales son definidos por un doble anclaje: uno temporal y otro espacial, que los unen con un territorio y una población a través de tradiciones de producción y consumo. El consumidor valora del queso su sabor y la posibilidad de recordar momentos, lugares y personas que forman parte de su identidad. A su vez, hay una serie de elementos en torno a estos principales, como el grado de preferencia, el origen del queso, su prestigio y el proceso productivo (Cervantes et al., 2017).

\section{Conclusiones}

Las empresas queseras en la subregión Ríos son microempresas familiares del sector privado. Entre los principales productos que elaboran se destaca el queso de poro, el tipo Oaxaca y la mantequilla, siendo el producto líder el queso de poro; el cual cuenta con marca colectiva, conocido y consumido localmente. Cuentan con mercados locales y regionales.

Pocas empresas realizan análisis de control de calidad a la leche o al producto terminado. La mayoría de las empresas utilizan la cera como material de empaque $y$, recientemente, algunas han comenzado a utilizar el empaque al vacío. No cuentan con procesos estandarizados ni documentados. Por ahora no emplean personal universitario; sin embargo, están dispuestos a realizar vinculación con instituciones educativas.

Aunque el gobierno mexicano ha apoyado mediante el decreto de leyes para la micro y pequeña empresa, así como la apertura de espacios como el Festival del Queso Artesanal, es importante que las empresas establezcan estrategias para definir las características de sus productos: desde la materia prima hasta los canales de distribución. De igual manera, es indispensable la asesoría para mejorar los procesos que involucren, principalmente, la inocuidad, el empaque del producto, condiciones de conservación y estandarización y documentación de procesos. 


\section{Referencias}

Arce, C.; Aranda, E.; Osorio, M.; González, R.; Díaz, P. y Hinojosa, J. (2017) Evaluación de parámetros productivos y reproductivos en un hato de doble propósito en Tabasco, México. Revista Mexicana de Ciencias Pecuarias, 8(1), 83-91.

Ayuso, G. y Castillo, M. (2017). Globalización y nostalgia. Cambios en la alimentación de familias yucatecas. Estudios Sociales, 27(50), 1-28.

Barba-Macías, E.; Rangel-Mendoza, J. y Ramos-Reyes, R. (2006). Clasificación de los humedales de Tabasco mediante sistemas de información geográfica. Universidad y Ciencia, Trópico Húmedo, 22(2), 101-110.

Barragán, E. y Ovando, P. (2015). Gestión cultural de un producto artesanal: queso Cotija región de origen. Segundo Encuentro Nacional de Gestión Cultural. Diversidad, tradición e innovación en la gestión cultural. Tlaquepaque, Jalisco.

Boscán, M.; Romero, J. y Sandrea, M. (2007). Fuentes de financiamiento utilizadas por las empresas productoras de derivados lácteos del estado Zulia. Revista de Ciencias Sociales, 13(1), 134-146.

Cano, M.; Bello, E. y Barba, E. (2011). Innovación social y capacidad de organización de las cooperativas pesqueras en el municipio de Balancán, Tabasco, México. Estudios Sociales, 20(39), 65-97.

Capdepont-Ballina, J. y Marín-Olán, P. (2014). La economía de Tabasco y su impacto en el crecimiento urbano de la ciudad de Villahermosa (1960- 2010). LiminaR, Estudios Sociales y Humanísticos. 12(1), 144-160.

Castañeda, T.; Boucher, F.; Sánchez, E. y Espinoza, A. (2009). La concentración de agroindustrias rurales de producción de quesos en el noroeste del Estado de México: un estudio de caracterización. Estudios sociales, 17(34), 74-109.

Castellot, R. (2014). ¿Productividad y competitividad? La micro y pequeña empresa en México ante la globalización. Horizontes de la Contaduría, 1; 48-61.

Castro-Georgana, V.; Díaz-Rodríguez, A. y Torres-Torres, B. (2005). Análisis de la calidad sani- 
taria de las queserías y los quesos en el estado de Tabasco en el período del 2002-2005. Salud en Tabasco, 13(1), 560-567.

Cervantes, F.; Patiño, A.; Cesín, A. y González, V. (2017). Innovando los estudios de mercado de los quesos artesanales. El valor simbólico del queso de poro. Estudios Sociales, 49(27), 67-91.

Correa, C.; Boucher, F. y Requier-Desjardins, D. (2006). ¿Cómo activar los sistemas agroalimentarios localizados en América Latina? un análisis comparativo. Agroalimentaria, 22; 17-27.

De la Cruz, A.; Romero, J.; Carrillo, J.; García, E.; Grether, R.; Sánchez, R. y Pérez, M. (2013). Brúquidos (Coleoptera: Bruchidae) del estado de Tabasco, México. Acta Zoológica Mexicana, 29(1), 1-95.

Díaz, M.; García, M.; Jiménez, J. y Villanueva, A. (2016). Inocuidad en alimentos tradicionales: el queso de Poro de Balancán como un caso de estudio. Estudios Sociales, 47; 87-111.

Estrada, F.; Barba, E. y Ramos, R. (2013). Cobertura temporal de los humedales en la cuenca del Usumacinta, Balancán, Tabasco, México. Universidad y Ciencia Trópico Húmedo, 29(2), 141-151.

Hernández, C.; Hernández, A.; Villegas, A. y Aguirre, E. (2011). El proceso socio-técnico de producción de Queso Añejo de Zacazonapan, Estado de México. Revista Mexicana de Ciencias Pecuarias, 2(2), 161-176.

Manjarrez, B.; Hernández, S.; De Jong, B.; Nahed, J.; De Dios, O. y Salvatierra, E. (2007). Configuración territorial y perspectivas de ordenamiento de la ganadería bovina en los municipios de Balancán y Tenosique, Tabasco. Investigaciones Geográficas, Boletín del Instituto de Geografía, 64; 90-115.

Queso de Poro Genuino de Balancán (2009). Queso de Poro, un producto genuino: logros y perspectivas. Diálogos, 31; 22-28.

Patlán, J.; Navarrete, D. y García, R. (2013). Perfil de competitividad y capital humano de empresas mexicanas basadas en la innovación. Negotium, 8(24), 109-140.

Poméon, T. y Cervantes, F. (2010). El sector lechero y quesero en México de 1990 a 2009: entre lo global y local. Reporte de Investigación, 89; 1-47. 
Rubio, I.; De Luna, J. y Ruiz-Sánchez, R. (2012). Propuesta de integración para la industrialización y comercialización de la leche en Tabasco. Universidad Autónoma Chapingo, México. Recuperado de http://www.chapingo.mx/zootecnia/assets/11ismael.pdf.

Salvador-Valencia, S. M. y Reyes-De la Cruz, C. (2011). Las Microempresas eje del Desarrollo Económico. El caso de Villahermosa, Centro, Tabasco. Memorias de la Semana de Divulgación y Video Científico 2011. Villahermosa. pp 275-278.

Tapiero - Cuellar, J.; Soleno, R.; Lozada, A.; Blandon, V.; Ramírez, K.; Rosero, M. y Rivas, Y. (2017). Evaluación de la vida útil de quesos semimaduros con recubrimientos comestibles utilizando aceite esencial de jengibre (Zingiber officinale) como agente antimicrobiano. Revista Colombiana de Investigaciones Agroindustriales, 4(1), 78-87.

Vargas-Uscategui, R.; Arenas-Clavijo, A. y Ramírez-Navas, J. (2017). Efecto del proceso de acidificación sobre el color de queso cottage. Agronomía Mesoamericana, 28(3), 677-690.

Villegas, A. y Cervantes, F. (2011). La genuinidad y tipicidad en la revalorización de los quesos artesanales mexicanos. Estudios Sociales, 19(38), 145-164. 